\title{
NORBERT ELIAS, RETRATO DE UN MARGINADO
}

\section{Helena Béjar}

Universidad Complutense de Madrid

«Los hombres llevan dentro de ellos, en lo que concierne a sus vidas, una intuición cuyo origen se remonta a los primeros momentos pasados con sus familias.» Estas palabras de Norbert Elias, al principio de la entrevista que constituye el grueso de Norbert Elias par lui-même, pueden servir de gozne entre la vida y la obra de este autor. Afirmar que los deseos dominantes de las gentes se configuran en la niñez, constituye toda una declaración teórica de principios. Por algo Elias reconoce en Freud su mayor influencia ${ }^{1}$, y gran parte de su opus magnum se dedica a las investigaciones psicogenéticas de la civilización occidental. Pero, además, en el caso de Elias, trabajos y días se entretejen de forma algo más que casual. Al menos, eso se deduce de la larga y apasionante entrevista originalmente publicada en un semanario holandés en 1984 y traducida al francés.

Certeza es lo que se desprende de sus primeros recuerdos en su Breslau natal, en una casa cuyos vastos espacios destinados a recibir visitas evoca el retrato del mundo aristocrático de La sociedad cortesana. Elias deriva esa primigenia seguridad de un padre «alemán, muy alemán», propietario de una peque-

${ }^{1}$ N. Elias, citado en J. Goudsblom, «The Sociology of Norbert Elias: Its Resonance and Significance», Theory, Culture and Society, 1987, vol. 4, núms. 2-3. 
ña fábrica textil, y una madre adaptada al modelo tradicional de diferencia armónica. "Era exactamente como en el Antiguo Régimen: uno no se podía imaginar que el mundo pudiera llegar a ser diferente» ${ }^{2}$.

Ni siquiera para un judío. Con una severidad que deja perplejos a los entrevistadores, Elias niega que, cuando niño, tuviera conciencia de pertenecer a la comunidad judía: "Lo siento, pero eso supone un nivel de conciencia que yo no tenía en aquella época.» Es precisamente la mirada sociológica la que inventa una reflexividad ubicua y no pocas veces artificial: «Me parece que gracias a las ciencias sociales estamos inclinados a explicar muchas más cosas que en la época donde no existía la ciencia social»" ${ }^{3}$.

El servicio militar en 1915 y la Primera Guerra Mundial constituirán el primer punto de inflexión de existencia tan segura "aunque aquí me falla la memoria». El Kaiser es una figura ridícula para Elias, que defiende su identificación con la cultura alemana sin por ello querer definirse como nacionalista.

Tras la guerra cursa estudios de filosofía y medicina, carrera que acaba abandonando. Es fácil rastrear la huella de tales estudios en la orientación biologista de muchos de sus escritos. Su insistencia en que nada hay en el sistema nervioso que refleje — tal como la tradición filosófica occidental sosteníauna división entre «lo exterior» y «lo interior» ${ }^{4}$ y su peculiar empeño en cuestionar los a priori kantianos (cuya naturaleza trascendental —y no psicológica, tal como él interpreta- parece no entender) le llevan a un fuerte enfrentamiento con su director de tesis. Sus alusiones a «un poder potencial mayor que el mío» reenvían a una noción eliasiana de poder como un concepto relacional y dinámico que entronca con su énfasis en la interdependencia entre los hombres.

Ante la ruina económica de la pequeña empresa paterna, el joven Elias se ve obligado a trabajar durante dos años en una fábrica. Con su habitual tendencia a positivizar todas sus experiencias (incluso los duros ejercicios militares le sirvieron - dice - para fortalecerse), Elias recuerda aquel período como un tiempo que le enseñó "cosas prácticas de la economía». Así, si el capataz explicaba su obsesión por el trabajo como "una caza por el contrato», él lo relaciona con «la fiebre de la lucha por el poder». Años depués conectará aquella "caza» con la concurrencia entre los nobles rivales en el proceso de estatalización.

En 1925 deja Breslau y se traslada a Heidelberg, meca de una sociología poderosamente influida por Weber, Simmel y Troeltsch, que compiten con la alargada sombra del materialismo histórico. En Heidelberg, Elias conoce, entre otros, a Alfred Weber y a Mannheim, de quien será ayudante. Aunque Stephen Mennell dice que Mannheim y Elias eran amigos, éste se detiene (en uno de los artículos que acompaña la entrevista de Elias par lui-même) en polemizar

N. Elias, Norbert Elias par lui-même, París: Fayard, 1991, p. 23.

3 Op. cit., p. 19.

4 S. Mennell, Norbert Elias (Civilization and the Human Self-Image), Oxford: Basil Blackwell, 1989, p. 7.

5 ELIAS, op. cit., p. 45. 
largamente con la sociología del conocimiento de Mannheim, así como en airear los detalles de la competencia académica de éste con Alfred Weber y, una vez en Londres, con Morris Gingsberg. El morbo del lector académico no se verá defraudado al ver que «los grandes» son también humanos.

El relato que Elias hace del Frankfurt de los años treinta (adonde se traslada con Mannheim para posteriormente trabajar en el Instituto de Investigación Social) es bastante difuso. Elias resalta el progresivo desplazamiento a la derecha de una clase media que se va embargando de una "amargura casi fanática ${ }^{6}$ con el aumento de poder de la clase trabajadora. Ante las evasivas de Elias a las insistentes preguntas de por qué partido votó, la tensión en la entrevista crece. Elias acaba respondiendo more sociologico: «... en la situación de la época, era evidente que el hecho de ir a votar o no ya no era esencial. Ello hubiera servido, como mucho, para descargarse en el plano emocional, nada más» ${ }^{7}$.

A principios de 1933 asiste, para ver el fenómeno del nazismo de cerca, a un mitin donde habla Hitler, corriendo el riesgo de que sus rasgos inequívocamente judíos le delaten. Cuando la enfervorizada multitud entona himnos, él mueve los labios. Poco después retira las fichas que comprometen a los componentes del Instituto, horas antes de una inspección de las SS. Es retenido durante unas horas: "Me zarandearon ¡a mí, Norbert Elias!». Un mes después se exilia en Francia.

Los dos años siguientes son especialmente duros, incluso de hambre. Sin posibilidad de hallar un empleo académico, Elias gasta el último dinero que le envía su padre en montar una pequeña fábrica de juguetes que pronto quebrará. En París conoce a Malraux, Gide y Koyré y nace su profunda admiración por la cultura francesa. En 1935 viaja a Inglaterra, donde permanecerá la mayor parte de su vida. En 1938 los padres de Elias van a Londres. Será la última vez que les vea, antes de morir en los campos de concentración nazis. A ellos está dedicado El proceso de civilización. Elias recuerda, emocionado, cómo su padre le dijo en aquella última conversación: "Jamás he hecho nada malo. ¿Qué me pueden hacer?»". Y es que los alemanes —según Elias- no advertían el ominoso peligro que les acechaba. En este sentido, pretender que existía una conciencia colectiva de amenaza no es sino «una proyección a posteriori» (p. 69), consecuencia de una excesiva implicación o "cercanía» con los acontecimientos que acaecieron después.

Durante esos primeros años en Londres trabaja apasionadamente en la British Library en El proceso de civilización. Sin empleo y sin dinero, Elias saca fuerzas de una pasión intelectual inquebrantable y verdaderamente emocionante. El Nouveau traité de civilité de Courtin despierta su interés por los manua-

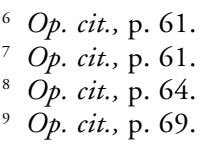


les de comportamiento y las maneras de las cortes europeas. Y, de nuevo, tras su entusiasmo teórico, asoma ese aplomo que le sostiene siempre: "Ese sentimiento de gran seguridad que experimenté en mi infancia explica mi perseverancia después, en la época en la que escribía mis libros y nadie me prestaba atención (...) no tenía una certeza absoluta, por supuesto, pero no dudaba de que mi obra sería un día reconocida como una contribución de calidad al saber de la humanidad ${ }^{10}$.

$\mathrm{Y}$ es que Elias posee una concepción redentora de la sociología. El teórico del «distanciamiento», una suerte de neutralidad axiológica o de reflexividad liberadora, alude una y otra vez a su misión, a una llamada interna destinada a destrozar mitos conceptuales y ocultaciones ideológicas: «Lo que quería era levantar el velo de las mitologías que ocultan nuestra visión de la sociedad a fin de que las gentes pudieran actuar mejor y de manera más razonable» ${ }^{11}$. La misión de un científico es atreverse a afrontar una verdad desmitificadora: «Debería haber más gente como yo que no teme lo que descubre (...) los hombres temen sorpresas desagradables si reflexionan sobre sí mismos con un poco de realismo. Freud, a su manera, quería descubrir las cosas como eran realmente, con independencia de lo que se hubiera dicho antes»" ${ }^{12}$.

Esa voluntad innovadora conceptual se refleja en la crítica que Elias hace a la dicotomía individuo/sociedad (que recorre el pensamiento moderno y culmina con el estructural-funcionalismo parsoniano, que Elias combate con la noción de configuración) o al determinismo económico de la realidad social por parte de la tradición marxista y su cosificada noción de poder (que él dinamiza a través de sus ideas de interdependencia y equilibrio de fuerzas) ${ }^{13}$. "Quería dar una idea de la sociedad que no fuera ideológica y lo conseguí»" ${ }^{14}$. Entregado al cometido de desvelar ideologías, elabora un aparato conceptual (compuesto por nociones como "configuración", "campo de fuerzas», "escalera de caracol», "paralelepípedo de poder», "enlace doble», etc.) carente de toda concreción temporal. Lo curioso es que dicho aparato tiene como sustrato una insistencia en el cambio y la interdependencia que se ofrece, sobre todo, como contrapropuesta al estatismo y solipsismo parsonianos ${ }^{15}$.

Elias se siente parte de una tradición que incluye a Comte, Darwin y Marx, esto es, del evolucionismo, aunque a lo largo de la entrevista nunca aluda abiertamente al mismo. Eso sí, se refiere a una "cadena de generaciones»

10 Op. cit., p. 24.

11 Op. cit., p. 50.

12 Op. cit., p. 64.

13 En relación a la oposición de Elias al marxismo y al funcionalismo, desde un análisis crítico de su propuesta teórica, puede verse H. BÉJAR, "La sociología de Norbert Elias: las cadenas del miedo", Revista Española de Investigaciones Sociológicas, núm. 56, octubre-diciembre 1991, pp. 61-82.

14 Op. cit., p. 50.

15 Al respecto, véase F. BOURdarias, «Norbert Elias: les techniques du regard», Sociétés, núm. 33, 1991, pp. 253-259. 
de la cual él se sabe un importante eslabón. En el último artículo de Elias par lui-même explicita su proyecto de fundar una teoría sociológica no ideologizada que las generaciones futuras puedan continuar: «La teoría del proceso de civilización y de la construcción del Estado, la teoría simbólica del saber y de las ciencias y, en un sentido más amplio, la teoría del proceso y de la configuración que me esforcé en elaborar no son ni marxistas ni liberales, ni socialistas ni conservadoras ${ }^{16}$. Asimismo, presenta el concepto de configuración como un intento de trascender lo que él considera estériles debates - metodológicos y sustantivos - entre individualismo y decisionismo, de un lado, y colectivismo y determinismo, de otro.

A veces, su propuesta aparece como una solución indiscutible cuyo cuestionamiento interpreta como «una resistencia contra una progresión hacia un nivel más elevado de conciencia ${ }^{17}$. Investido de una nueva verdad — paradójicamente, desmitificadora-, afirma: "uno no puede enseñar a ver a los ciegos...». Esta soberbia intelectual, que puede resultar un punto irritante, se acompaña - y se compensa- de ese ya mencionado entusiasmo por la empresa intelectual que humaniza al personaje: «Siempre me ha espantado ver la cantidad de gente que se desalienta en nuestros días, como si nada quedara por hacer, mientras hay tanto por hacer y tanta gente que hace cualquier cosa y que está corrompida intelectualmente... ${ }^{18}$. Esta tristeza desencantada contrasta con su absoluta entrega al trabajo teórico durante la gestación de El proceso de civilización.

«Escribí este libro sin darme cuenta de que no interesaba en absoluto a los demás. Por mi parte, hallaba el tema absolutamente apasionante» ${ }^{19}$. Mennell distingue tres acepciones en la noción de civilización. En primer lugar, ésta se refiere a un proceso de formación de la personalidad cuya hipótesis, el avance de la contención emocional desde un heterocontrol social a un control internalizado, pretende oponerse a la psicología social imperante en los años treinta. En segundo lugar, la civilización es un modelo de conducta (consistente en la doma de la violencia, la constricción de todo exceso sentimental y la moralización de conductas otrora "naturales») que corre paralelo a la formación del estado moderno. En tercer lugar, es un macroproceso que engloba a toda la humanidad.

Sin duda, la aportación más preclara de Elias a la historia de la sociología es la imbricación en su obra entre los niveles «micro» y «macro», esto es, entre la urdimbre de las intenciones individuales y los procesos sociohistóricos de larga duración. Sin embargo, más que hacia lo "micro" (el análisis de los manuales de comportamiento de la época clásica y los códigos moralizantes que legitiman la mudanza de la «economía afectiva», o la primorosa descrip-

16 Op. cit., p. 165.

17 Op. cit., p. 167.

18 Op. cit., p. 96.

19 Op. cit., p. 73. 
ción de la socialidad de la corte del Rey Sol en La sociedad cortesana), los dardos contra Elias se dirigen a su vertiente "macro», a su intento de explicar los procesos no planeados de cambio. Por ello, en las evaluaciones - necesariamente parciales - a que son sometidos todos los autores, en el caso de Elias, no suelen tenerse en cuenta sus intuiciones acerca de temas de gran actualidad en la teoría sociológica. Así, la importancia de la reflexividad en el conocimiento y en la vida social ${ }^{20}$. Pero será el gran proyecto eliasiano y su orientación de larga duración el blanco de sus detractores.

Siempre al quite de las críticas a su maestro, Mennell hace de Norbert Elias: Civilization and the Human Self-Image un espejo fidelísimo y devoto del objeto que estudia. Veamos brevemente algunas de las líneas de ataque. En primer lugar, Mennell discute el supuesto determinismo del mecanismo de monopolio, clave en el proceso civilizatorio. Ante la acusación de determinismo demográfico (los nobles feudales compiten por una tierra que se hace más y más escasa, y esta carencia explica migraciones, conquistas, cruzadas, urbanización y, a la postre, cortesanización de las conductas), Mennell no hace sino repetir el argumento de Elias: «En este período particular, la tierra era el medio de producción más importante y la fuente clave de poder (...) la población creciente tenía un efecto más simple y directo en la lucha por la tierra que en una sociedad más compleja $»^{21}$. Contra el reproche de mantener un mecanicismo pasado de moda (de hecho, en la explicación del mecanismo de monopolio Elias rememora — sin mencionarla- la explicación durkheimiana de la urbanización), Mennell recuerda las reticencias de Elias a referirse a leyes sociológicas y su afinidad con explicaciones de carácter más probabilístico. Lo malo es que Mennell no fundamenta su contracrítica con cita alguna. Pero, de entre los argumentos contra las hipótesis de Elias, Mennell se concentra especialmente en el frente que ofrecen los relativistas culturales que — según él— pretenden falsar por completo las hipótesis eliasianas y «buscan bloquear toda investigación ulterior de los temas que la teoría ofrece» ${ }^{22}$.

En el capítulo titulado «La humanidad como un todo», Mennell reconoce que la civilización, como proceso de formación de la personalidad, como doma de las maneras o como sinónimo de diferenciación cultural, es un universal. Elias dijo, y dijo bien: todos los hombres tienen que aprender a controlar sus emociones. Otra cosa es que tal aprendizaje varíe de sociedad a sociedad ${ }^{23}$. En el

${ }^{20}$ Los temas de la no intencionalidad y de la reflexividad de lo social han generado una bibliografía que no puedo señalar aquí. Como muestra, véanse R. BOUDON, Efectos perversos y orden social, México: Premiá, 1980, y La place du désordre, París: PUF, 1984; E. LAMO DE ESPINOSA, La sociedad reflexiva, Madrid: CIS, 1990, y R. RAMOS, «Una aproximación a las paradojas de la acción social», en E. Lamo de Espinosa y J. E. Rodríguez Ibáñez (eds.), Problemas de teoría social contemporánea, Madrid: Centro de Investigaciones Sociológicas, 1994.

${ }^{21}$ S. Mennell, Norbert Elias (Civilization and the Human Self-Image), Oxford: Basil Blackwell, 1989, p. 64.

22 Mennell, op. cit., p. 250.

${ }^{23}$ Op. cit., p. 207. 
siguiente capítulo, Mennell la emprende contra el agnosticismo desarrollista (developmental agnosticism) que se ha extendido en la ciencia social del siglo XX.

¿Elias unilineal? El proceso civilizatorio no tiene ni origen (Elias negó siempre un "punto cero») ni meta a la que aspirar. Comte puede ser uno de los modelos de Elias (como "destructor de mitos», le dedica todo un capítulo de Sociología fundamental), pero éste no tiene en mente sociedad positiva alguna como culminación de un progreso indudable. Es más, en sus últimos escritos Elias se torna sombrío en sus predicciones sobre el precario equilibrio de armamento -en Humana Conditio_- así como con el individualismo rampante de nuestro fin de siglo —en La soledad de los moribundos-. Por si fuera poco, Mennel saca a colación la imagen de la «espiral» o "escalera de caracol» que, a cada vuelta, incorpora nuevos elementos. Lástima que dichas expresiones eliasianas sean meras metáforas sin un desarrollo suficiente que permita una conceptualización global del proceso de civilización.

¿Elias racista? Absurda acusación, replica Mennell, puesto que la civilización no es algo natural, sino un proceso social e histórico. Curiosamente, Mennell no parece caer en la cuenta de que tal afirmación es contradictoria con la idea de que el autocontrol es un universal: "todos los seres humanos tienen que aprender a controlar sus emociones, "impulsos" $\mathrm{e}$ "instintos". No son “civilizados" por naturaleza, sino civilizables por naturaleza»" ${ }^{24}$.

¿Elias etnocéntrico? En absoluto, al menos en el sentido del evolucionismo clásico, que pretende poner todas las culturas en un mismo eje de desarrollo. Elias, por el contrario, sólo pretende estudiar una secuencia de cambios en una cultura concreta - la europea y, se podría añadir, la francesa- y en una época específica —-desde la Alta Edad Media al inicio de la Ilustración-.

Así, entre el evolucionismo de la antropología social clásica y el estructuralismo de Lévy-Strauss, Elias adopta un punto intermedio. El mismo, se diría, que Mennell, ante la dificultad de defender a su maestro de contribuir a un neoevolucionismo — con todos los matices que se quiera- sobre el que descansa su opus magnum: "... se puede decir que en sus primeros libros sobre la formación de la civilización europea, el equilibrio de sus preocupaciones se inclinaba ligeramente hacia la cara "multilinear" y particularista y que, después, en sus trabajos sobre los procesos de civilización al nivel de la humanidad como método se inclina ligeramente del otro lado. Pero siempre recalcó que el desarrollo social supone simultáneamente (...) regresiones y progresiones» ${ }^{25}$. El empeño de Mennell en salvar las hipótesis y, simultáneamente, en hallar un punto arquimédico entre evolucionismo y "apertura» (en la acertada expresión de Allan Bloom) conduce, como poco, a la perplejidad. Cabe preguntarse por qué no aceptar, sin sonrojo, que el evolucionismo de Elias no desmerece la grandeza de El proceso de civilización. Es decir, burlar el trapo del relativismo.

24 Op. cit., p. 206.

25 Op. cit., p. 236. 
Pero volvamos al relato de la vida de Elias. Durante tres años, un comité de ayuda a refugiados judíos le ayuda económicamente en la redacción de $E l$ proceso de civilización, cuya primera edición —en alemán - financia el padre del autor. Se publica en Suiza. Las ventas y la difusión del libro serán mínimas. Elias permanece en Inglaterra desde 1935 a 1975, estancia interrumpida por un breve período en Ghana —entre 1962 y 1964_, seguramente por razones económicas, aunque él se jacta de haber tenido allí la ocasión de estudiar el arte africano. En el departamento de sociología de la Universidad de Leicester (por el que pasaron Albrow, Dunning, Giddens, Goldthorpe y Mouzelis) no logra un empleo remunerado hasta 1954, puesto que le proporciona Neustadt, también refugiado de guerra. Consciente de su marginación, le sigue sosteniendo su vocación: «Siempre me creí capaz de hacer algo relativamente importante. Esa fe jamás flaqueó $»^{26}$.

Empero, pasa durante un tiempo por el diván del psicoanalista porque «sufría por no lograr producir teniendo muchas ideas»" ${ }^{27}$. Sin embargo, no reconoce dicha terapia como una experiencia determinante en su vida: «en tanto que se pueda, uno debería ayudarse a sí mismo $»^{28}$. (Curiosa afirmación, precisamente en boca de un autor que propone una noción - la de configuracióncuya materia básica es la interdependencia y que critica, además, las implicaciones teóricas y morales del homo clausus.) Elias es uno de los fundadores de la Sociedad Analítica de Grupo. El mismo dirige grupos de terapia.

Su profundo sentimiento de marginación no se mitigará hasta que no se marche de Inglaterra. Mennell alude a la amargura de Elias al recordar que en Leicester no dejó discípulos. Cosa muy distinta sucede en Holanda, en cuyas universidades pasa largas temporadas hasta su muerte, y que hoy constituyen el núcleo de estudios alrededor de la llamada sociología figuracional. Recordando de nuevo a Freud, Elias sabe que, como él, ha nadado siempre a contracorriente y que el estudio de la "organización discrónica de la sucesión", de los procesos sociohistóricos de larga duración no planificados, se oponía al hegemónico estructural-funcionalismo. Tras su sociología histórica —en mi opinión, lo mejor de su obra - se embarca a partir de los años setenta en el proyecto de la sociología figurativa y una peculiar sociología del conocimiento que no obtienen predicamento alguno. No es extraño que Elias se refiera a un fantasma (que proviene de la oscura época de Leicester) que expresa su túnel particular: «Hablo por teléfono y la voz, al otro lado del hilo, me dice: "¿Puede hablar un poco más alto? No le oigo.” Me pongo a gritar y la voz repite constantemente: “Podría hablar un poco más fuerte? No le oigo” ${ }^{29}$. Fuera de modas y escuelas,

${ }^{26}$ ELIAS, op. cit., p. 86.

27 Elias, op. cit., p. 83.

28 Op. cit., p. 84.

29 Op. cit., p. 93. 
insiste en la entrevista en la necesidad de mantener la integridad intelectual y de resistir las imposturas de las corrientes dominantes de pensamiento. Hoy, cuando la existencia de la propia teoría sociológica se cuestiona y muchos se arroban ante los cantos del homo oeconomicus, la postura de Elias cobra especial valor.

Hablaba, al principio de estas páginas, de la profunda imbricación entre su vida y su obra. En 1965, Elias publicaba, junto con John Scotson, The Established and the Outsiders. A mi juicio, dicho libro no es sólo, como indica su subtítulo, una investigación sobre los problemas de una comunidad. En él se perfilan vivencias propias.

Lo que distingue a los «establecidos» de los «marginados» son los «recursos de poder" que cada uno de ellos dispone. A mayor desigualdad de poder — político, económico, afectivo, etc.—, menos realismo y más distorsión en la percepción de los establecidos. Individuos y grupos crean una "visión en blanco y negro" basada en el juicio de una "minoría de lo mejor» a la cual se envidia y una "minoría de lo peor» a la que se desprecia. La autoimagen y la visión del otro dependen, pues, del «diferencial de poder» y de la autoconfianza de los miembros del grupo. Este modelo se puede aplicar a varios fenómenos.

Por ejemplo, a los judíos, en tanto que grupo históricamente marginado. Elias rememora cómo ya en el colegio, cuando dijo que quería ser profesor de mayor $^{30}$, sus compañeros le contestaron que, como judío, nunca podría serlo. En «Notas sobre los judíos como participantes en una relación establecidosmarginados» —en Elias par lui-même- sí reconoce que su condición de judío alemán ha influido poderosamente en su autoconciencia y en su pensamiento. Recuerda la comunidad judía de su infancia como una sociedad que disimulaba su exclusión social: "se era un hombre de segundo orden, pero uno no se consideraba como tal $»^{31}$. Marginados en la universidad, la diplomacia y el alto funcionariado, los judíos buscan su integración en los ámbitos de la economía y de la cultura.

La polaridad integrados/marginados revierte en el grado de conocimiento que cada elemento del par tiene del otro. Así ocurre en el amor, donde el amante conoce o se propone conocer al amado mucho más que éste a aquél, menos interesado en las peculiaridades del amante, a quien se da por supuesto, por algo seguro y, por tanto, poco merecedor de investigación alguna. Cosa parecida sucede con la mayor "comprensión" de las mujeres — respecto a los hombres - debida a un diferencial de poder atribuido tradicionalmente a la «intuición femenina» ${ }^{32}$.

Elias aplica, asimismo, el modelo de los established/outsiders para explicar la

30 «Es como si Elias hubiese sido siempre viejo.» Vrij Nederland, 1 de diciembre de 1984, cit. en S. MENNELL, p. 3.

31 Op. cit., p. 155.

32 Para un análisis de las relaciones entre los sexos, dentro de un enfoque eliasiano de los códigos de conducta actuales, véase H. BÉJAR, «La ordenación de los placeres», en E. Gil Calvo (comp.), Los placeres: éxtasis, prohibición y templanza, Barcelona: Tusquets, 1992. 
violencia de los hooligans. Estos llamativos aficionados a los deportes de masas hacen gala de una violencia expresiva que se corresponde a unos vínculos segmentales o funcionales - propios de una solidaridad mecánica- que se fortalecen con el apoyo a "los suyos» (we-group o established) y de la hostilidad con los rivales (they-group o outsiders). Tal como resume Mennell: «La moderna estructura de interdependencias sociales ha aumentado el poder de los jóvenes machos, de origen decididamente marginal, para que expresen normas que entren en conflicto con las de los grupos establecidos ${ }^{33}$.

Para Elias, la violencia que se da en el deporte actual (tanto en su forma expresiva, la de los gamberros, como en su vertiente instrumental, gerenciada por la competencia profesional que promueven las corporaciones deportivas) es propia de una sociedad pacificada. A través del deporte se expresan tanto los desmanes de las tribus urbanas como la ritualización que conlleva el renacimiento de la religión civil ${ }^{34}$. Contra esta supuesta pacificación claman los críticos de la teoría de la civilización, que defienden una creciente y ubicua barbarie. Mennell ve en "el argumento de la barbarización», «el argumento de las civilizaciones sin estado" (cuyos defensores contestan el paralelismo que hace Elias entre civilización y estatización) y «el argumento de la sociedad permisiva» (que opone a la tesis eliasiana del progresivo autocontrol una creciente informalización del comportamiento) líneas de análisis abiertas para investigaciones futuras. Ni que decir tiene que Mennell, en el tono hagiográfico que recorre su excelente libro, se las arregla para hacer encajar todos sus argumentos (con excepción del más duro, el del relativismo cultural, más arriba comentado) con la obra de Elias.

En lo que a la barbarización se refiere, recuerda cómo Elias señalaba que la civilización podría sufrir regresiones e incluso quebrarse. Es más, dice, mientras que la violencia entre estados va en aumento, la pacificación intraestatal decrece. De nuevo, la hipótesis está salvada. Lástima que Elias no viviera para ver cómo la pacificación ha alcanzado las relaciones interestatales. Al menos - lo que no es poco- entre las grandes potencias. El temor que destilan las páginas de Humana Conditio (Consideraciones en torno al futuro de la humanidad) se hubiera templado.

En Mozart (Sociología de un genio) confluyen varios de los temas más eliasianos, como la naturaleza no planeada de los procesos sociales, la polaridad establecidos/marginados y la necesidad de distanciamiento. Es difícil no adivinar a lo largo de sus páginas cierta proyección personal en el tratamiento de la figura de Mozart.

33 Op. cit., p. 157.

${ }^{34}$ Véase, al respecto, S. Giner, "Religión civil», Revista Española de Investigaciones Sociológicas, núm. 61, enero-marzo 1993. 
Los editores advierten sobre el carácter disperso e inacabado de estas páginas, que forman parte de un proyecto que truncó la muerte de Elias. El bosquejo y las notas descubren una doble perspectiva. En primer lugar, la explicación del "problema sociológico»: la figura de Mozart como expresión de la transición del arte artesanal al arte artístico. En segundo lugar, el "problema psicológico» en torno al arte como sublimación (noción en torno a la cual Elias se sume en un auténtico batiburrillo conceptual) de las carencias del músico.

Mozart quiere analizar el destino de las personas dentro de los grandes desarrollos históricos. La vida de aquél revela un microproceso dentro de un macroproceso de cambio general: «El destino de Mozart es una muestra conmovedora de un hombre que (...) fue a parar a un remolino de un proceso social no planificado»"35. Tal como en Elias par lui-même, Elias repite que "las líneas volitivas fijas de las personas» se configuran en la niñez gracias - o a veces por desgracia- a la convivencia con otras personas. Mozart poseía «una secreta solicitud de amor y afecto" insatisfecha tanto en su vida privada como en su carrera. En aquélla se debatía entre dos figuras de autoridad: un padre autoritario que trazó un férreo plan para su hijo prodigio y una esposa alocada e infiel. Tampoco su vida pública fue como él hubiera deseado, tanto por su peculiar rebeldía como por el hecho de adelantarse a su tiempo.

El modelo de arte artesanal era propio de sociedades no estatalizadas. Elias esboza una relación entre la fecundidad de la música en Alemania e Italia y su falta de integración nacional. En este sentido, el desarrollo de aquélla se vincularía a la fuerte competencia entre las múltiples cortes que recorrían los artistas. La posición de un músico era entonces la de un artesano empleado por el señor de la corte. Tenía que crear productos elegantes y entretenidos, al gusto del patrono que le empleara. Su fantasía estaba subordinada al canon del poderoso cliente. Cuando, en El rapto del serrallo, Mozart descentra a los cantantes y entrelaza las voces humanas con el sonido de los instrumentos estableciendo «una traslación en el equilibrio de poder», sus innovaciones no fueron bien recibidas. El emperador José II se aburrió ante tal alteración de la estructura operística clásica: «Tiene demasiadas notas», dijo.

El arte artesanal, por encargo, no era individualizado. No existía la noción de un estilo propio. Dicha configuración - la misma que Elias disecciona en La sociedad cortesana - se fundaba en una profunda distancia social y desigualdad de poder. El músico era un «cortesano servil» que se debía a su protector. Por ello, la desobediencia de Mozart a los dictados del obispo de Salzsburgo son inauditos para Leopoldo Mozart. La osadía de Wolfgang entrañará una amenaza fatal para su existencia social.

El padre de Mozart, presa de un resentimiento social que anida junto a un orgullo maltrecho, reprende a su hijo una y otra vez. La necesidad de llenar de sentido su vida a través de Amadeus pugna con el deseo de amor filial. En el

35 N. Elias, Mozart (Sociología de un genio), Barcelona: Península, 1991, p. 55. 
tiránico trazado vital de padre a hijo, aquél «no tenía otra alternativa». Como no la tenía Mozart, necesitado tanto del amor paterno como de su afirmación personal. Reencontramos aquí los temas de la ambivalencia y la interdependencia en los vínculos sociales, así como la comprensión de las figuras individuales dentro de grandes cadenas de acontecimientos que los hombres no pueden controlar.

La ambivalencia de Mozart es personal y social. Humillado por las afrentas que recibe de sus superiores desea, sin embargo, pertenecer a esa nobleza que le excluye por su oficio y su personalidad. En una carta, la emperatriz María Teresa advierte al archiduque de Milán sobre el carácter superfluo de sujetos como Mozart: «Si os divierte, no quiero privaros de ello. Lo que digo es que no hay que cargarse de gente inútil... Esta gente que viaja por todo el mundo como pedigüeños degrada el servicio de la corte» ${ }^{36}$.

Incapaz de comprender la estructura interna de la sociedad cortesana, Mozart — como la mayor parte de los actores sociales - veló la realidad de «deseos y fantasías" ${ }^{37}$. "En todo momento era una batalla personal y en esos términos se mantuvo siempre. Y ése fue uno de los motivos por los cuales tuvo que perderla ${ }^{38}$. Esta extrema implicación personal se relaciona con la necesidad de distanciamiento, tema crucial en la sociología del conocimiento de Elias.

El distanciamiento permite orientar las acciones de manera realista y, a veces, modificar la situación. Para Elias, el detour behaviour es un elemento esencial de la racionalidad. Está relacionado tanto con el progreso de la ciencia - a nivel macro - como con el del autocontrol - a nivel micro-. A este respecto el distanciamiento es un muro de contención de la inseguridad. Tal como recoge Mennell, «una extrema inseguridad ante los riesgos de la vida cara a las fuerzas incontrolables impide que la gente realice un desvío, a través del distanciamiento, necesario para desarrollar un conocimiento y unas explicaciones de tipo impersonal ${ }^{39}$. La inseguridad — afectiva y de clase- que sufría Mozart aumentaba su vulnerabilidad y su incomprensión de un proceso social que era incapaz de comprender y del que se sentía preso.

Su actitud con el arzobispo de Salzsburgo, para quien Mozart era no sólo su organista, sino su ayuda de cámara, es más propia del llamado arte artístico que del artesanal. Mozart se comportaba como un genio avant-la-lettre. La figura del genio - cuyo paradigma musical es Beethoven- se inscribe, entre otras condiciones sociales específicas, dentro de un mercado de compradores anónimos ${ }^{40}$. Tal mercado (que moviliza a los consumidores a través de mediadores tales como los editores o los empresarios) permite un equilibrio de poder

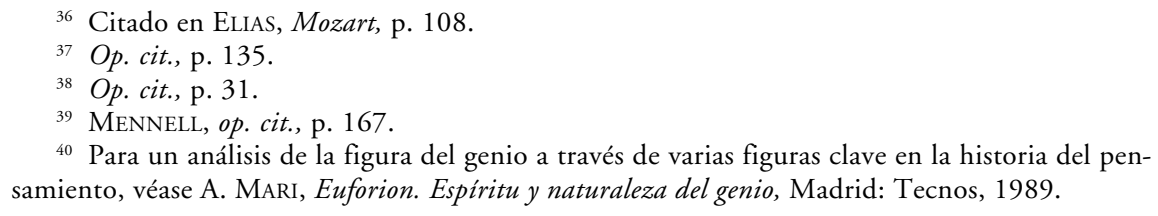

40 Para un análisis de la figura del genio a través de varias figuras clave en la historia del pensamiento, véase A. MARI, Euforion. Espiritu y naturaleza del genio, Madrid: Tecnos, 1989. 
que se inclina a favor de los productores de arte. Si el mundo aristocrático se regía por relaciones jerárquicas donde la dependencia se aúna con la seguridad que da la protección, el orden democrático entraña vínculos igualitarios donde la progresiva autonomía - del artista, en el caso que nos ocupa- se engarzará con la inseguridad económica.

Es una pena que, en Mozart, Elias no desarrollara la oposición entre el arte cortesano y el arte individualizado más allá de unas notas dispersas, y que prime la dimensión psicológica sobre la sociológica. A este respecto hay que resaltar que, cuando analiza la difícil relación del músico con el arzobispo de Salzsburgo, haga un excesivo hincapié en los conflictos internos de Mozart, cuya autoestima iba decreciendo con los años.

Elias apunta el interés de estudiar el sentido del humor de Amadeus, burdo, infantil y escatológico. La "coprofilia verbal de Mozart» expresa no sólo una fase histórica de baja contención de los impulsos - a nivel sociogenético-, sino también un retorno de lo reprimido junto con una personal rebelión contra la autoridad - a nivel psicogenético-. En este sentido, el humor es una suerte de "fuga de ideas»"1 que esconde un vacío vital y un creciente desconsuelo. En una carta a su hermana, Wolfgang escribe: "A propósito, ¿sabes la historia que ocurrió aquí? Ahora te la cuento: salíamos hoy de casa del conde Firmian para regresar a la nuestra y cuando llegamos a nuestra callejuela, abrimos la puerta y ¿qué crees que pasó? Pues que entramos. Que te vaya bien, mi pequeño pulmón, mil besos, hígado mío, y me despido como siempre, mi estómago, tu hermano que no es digno de ti, Wolfgang. Por favor, por favor, querida hermana mía, que me pica, ráscame $»^{42}$.

Diríase que, al final de sus días, Mozart no había satisfecho el deseo dominante de su vida: ser querido. En la voluntad de morir que le atribuye Elias se esconde una profunda pérdida del sentido existencial. Su grandiosa capacidad de sublimación no pudo hurtarle la infelicidad, horizonte de todo outsider: «Las personas que adoptan una posición marginal respecto a determinados grupos privilegiados, pero que se consideran iguales o mejores que ellos por su disposición personal o a veces por una alta autoestima, se defienden de la degradación a la que están expuestos mediante la amargura ${ }^{43}$. Incapaz de distanciamiento y carente de sentido alguno de misión, Mozart pertenece, no obstante, a una gran cadena de generaciones. Como cree de sí mismo el autor de esta biografía.

Norbert Elias, con su concepción redentora de la sociología, quiso obviar la búsqueda de la Buena Sociedad. Frente a la carga ideológica tanto del dina-

41 Elias, op. cit., p. 152.

${ }^{42}$ Citado en Elias, Mozart, p. 110.

43 Op. cit., p. 45. 
mismo histórico marxista como del conservadurismo del estructural-funcionalista, planteó un modo de pensar la sociedad libre de mitos. Sin embargo, cabe pensar que tan grandioso proyecto no se cumple sino en la elaboración de una batería de conceptos — de los cuales puede destacarse el de configuraciónque no forman un conjunto lo suficientemente cohesionado como para oponerse a las Grandes Teorías que combate. Al cabo, la pasión intelectual de Elias —atestiguada en los libros más arriba mencionados- se expresa sobre todo en su voluntad de unir el análisis micro y macro del avance del autocontrol. Es en la poderosa descripción de los procesos no planeados del cambio social donde Elias deja su impronta sociológica. Dicha empresa le hace merecedor de un puesto de honor en la teoría sociológica contemporánea y, así, trascender su condición de eterno marginado.

\section{RESUMEN}

El presente ensayo es una suerte de introducción a la figura de Norbert Elias, en un plano tanto personal como intelectual. En este sentido, se rememora su vida y su empresa teórica, haciendo hincapié en su totalizante proyecto neoevolucionista. Por otra parte, el personaje de Mozart —al que dedica su última obra — sirve para resaltar la condición de marginado que recorre la existencia de este autor. Durante un largo tiempo olvidado, ha sido al fin recuperado al hilo del interés teórico suscitado por la sociología histórica en relación a la explicación del cambio social.

\section{ABSTRACT}

This essay can be considered an introduction to Norbert Elias, both on a personal and an intellectual level. Elias' life and intellectual project are revised, especially his grand neoevolutionist sociology. On the other hand, Mozart — who was the subject of his last work — serves as a character which highlights Elias' nature as an outsider. His unjustly overlooked work has regained interest along with the contributions of historical sociology in the study of social change. 